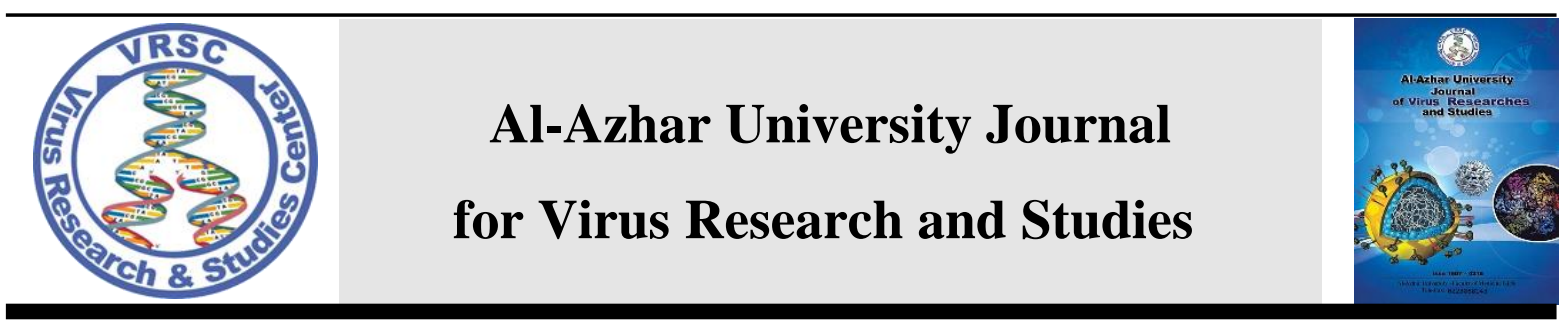

\title{
Genotypic and Phenotypic Screening of Carbapenemase Mediated Resistance in Klebsiella pneumoniae Clinical Isolates: A Hospital Based Study.
}

\author{
Haneya A.A. Anani*1, Asmaa A. El Madbouly², Azza A. Althoqapy ${ }^{1}$ and Omnia A. El- \\ Dydamoni $^{1}$
}
${ }^{1}$ Microbiology and Immunology, Faculty of Medicine (for girls), Al-Azhar University, Cairo, Egypt.

${ }^{2}$ Clinical Pathology, Faculty of Medicine (for girls), Al-Azhar University, Cairo, Egypt.

*E-mail: haneyaanani.micro@azhar.edu.eg

\begin{abstract}
Carbapenem-resistant Klebsiella pneumoniae (CRKP) has been reported increasingly all over the world and it is usually resistant to multiple antimicrobial agents. Is to detect the frequency and distribution of carbapenemase genes in CRKP at Al-Zahraa University hospital, Cairo, Egypt, and to compare between the performance of Rapidec Carba NP and modified carbapenemase inactivation (mCIM) phenotypic methods for early and reliable recovery of carbapenemase production in Klebsiella. pneumoniae (KP) clinical isolates. Sixty $K$. pneumoniae isolates were recovered in our Microbiology laboratory. Carbapenemase production by these isolates was assessed using antimicrobial sensitivity testing, Rapidec Carba NP, modified carbapenemase inactivation methods (mCIM) and detection of carbapenemase genes by PCR. CRKP was implicated in several types of healthcare associated infections with frequency of 46.7 (28/60). The frequency of carbapenemase genes was 13 (54.2) for blaNDM, 7 (29.2\%) for blaOXA-48 and 1(4.1\%) for blaKPC. 3 (12.5\%) isolates carried both blaNDM and blaOXA-48. All isolates were negative for blaIMP, the sensitivity and specificity of Rapidec Carba NP test were (100\%) and $(91.7 \%)$ respectively, while sensitivity and specificity of $\mathrm{mCIM}$ were $(66.7 \%)$ and $(88.9 \%)$ respectively, in comparison to the PCR test which is used as the gold standard test. The Carbapenem resistance is frequently encountered in K. pneumoniae isolates at our hospital. Rapidec carba NP and mCIM tests have high performance and applicability in detection of carbapenemases. However, The Rapidec carba NP was found to be rapid and with higher sensitivity.
\end{abstract}

Keywords: Klebsiella pneumoniae, Carbapenem-resistance, Rapidec Carba NP, Carbapenemes Inactivation Method, Carbapenemase genes. 


\section{Introduction}

Klebsiella pneumoniae (K. pneumoniae), is a significant frequently encountered nosocomial pathogen. Multidrugresistant $K$. pneumoniae (MDRKP) is considered a dangerous and urgent threat in clinical settings [1]. Carbapenems were considered a reliable and effective antibiotic against $K$. pneumonia, but during the last 10 years, carbapenemaseproducing $K$. pneumoniae (CPKP) incidence was dramatically elevated worldwide [2]. The Ambler carbapenemase classes A (KPC), B (VIM, IMP, NDM) and D (OXA-48) are the most significant carbapenemases in clinical settings. They are frequently detected in $K$. pneumoniae isolates associated with life threatening hospital acquired infections [3].

Consistent and rapid identification of CRKP is necessary for therapeutic management of patients, for antibiotic stewardship practice and for infection control to ban the dispersal of these multidrug-resistant (MDR) bacteria $[4,5]$.

Numerous procedures are present for rapid detection of CRKP as immunochromatographic assays, molecular techniques and matrix-assisted laser desorption ionization-time of flight (MALDI-TOF) technology, but these techniques are not routinely available in microbiology laboratory and also require significant expertise and high cost [6,7].

Carba NP test was suggested by the Clinical Laboratory Standard Institute (CLSI), 2012 for the recovery of carbapenemases in Gram-negative bacilli, but the reagent preparation required for the in-house test is sophisticated and the reagents are not stable for long duration, decreasing its clinical applicability [8]. Rapidec carba NP test is a commercially available carba NP kit that was designed for speedy detection of different carbapenemases [9].
In 2017, CLSI has suggested the modified carbapenem inactivation method (mCIM) for the recovery of carbapenemases in $P$. aeruginosa and Enterobacteriaceae [8].

The purpose of our study is to identify the frequency of CRKP in our hospital setting, their carbapenemase gene profile and to compare the performance of Rapidec Carba NP and modified carbapenem inactivation (mCIM) phenotypic methods to settle a screening phenotypic tool for detection of these isolates

\section{Materials and Methods}

\subsection{Study design:}

This is a hospital based cross-sectional study that was done on sixty isolates of $K$. pneumoniae, isolated from 315 clinical specimens provided to Microbiology laboratory of Al-Zahraa University Hospital, Cairo, Egypt for routine culture and antibiotic sensitivity testing in the period from March to August 2020.

This study has been approved by the Research Ethics Committee of the Faculty of Medicine for Girls, AL-Azhar University, Cairo, Egypt (approval no. 20192287) and a waving from obtaining an informed patient consent was approved by the Committee.

\subsection{Phenotypic identification of isolates and detection of carbapenem resistance:}

\subsubsection{Microbiological identification}

Isolates have been identified as $K$. pneumoniae depending on Gram staining, characteristic colonial morphology, and routine biochemical reactions.

\subsubsection{Antimicrobial sensitivity Testing:}

Antibiotic sensitivity testing was done implementing the Kirby-Baur disk diffusion method in accordance with the CLSI [10]. The K. pneumoniae isolates were tested against ampicillin $(10 \mu \mathrm{g})$, ampicillin/sulbactam $\quad(10 / 10 \mu \mathrm{g})$, amoxicillin/clavunate $\quad(20 / 10 \mu \mathrm{g})$, 
piperacillin $\quad(100 \mu \mathrm{g}), \quad$ pipracillintazobactam $(100 / 10 \mu \mathrm{g})$, cefoperozone $(75 \mu \mathrm{g})$, ceftazidime $(30 \mu \mathrm{g})$, cefepime $(30 \mu \mathrm{g})$, cefotaxime $(30 \mu \mathrm{g})$, norfloxacin $(10 \mu \mathrm{g})$, ciprofloxacin $(5 \mu \mathrm{g})$, cefoxitin $(30 \mu \mathrm{g})$, amikacin $(30 \mu \mathrm{g})$, gentamicin $(10 \mu \mathrm{g})$, meropenem $(10 \mu \mathrm{g})$, and ertapenem $(10 \mu \mathrm{g})$. CRKP was determined as isolated $K$. pneumoniae strains with resistance to at least one of the carbapenem drugs. As a quality control, the $K$. pneumoniae ATCC700603 strain was used for the antibiotic sensitivity testing.

\subsubsection{Double disk synergy method for confirmation of production of extended spectrum:}

beta lactamase (ESBL): the resistant isolates to ceftazidime and or cefotaxime using disk diffusion technique were confirmed for ESBL production by the double disk synergy testing using cefotaxime /ceftazidime/clavulanic acid inhibitory disk. It was considered positive for ESBL if there is extension of the edge of the inhibition zone of ceftazidime/ cefotaxime on the side exposed to the disc containing clavulanic acid [11].

\subsubsection{AmpC disk test:}

According to disk diffusion results, isolates with cefoxitin resistance were confirmed for ampC production by ampC disk test [12].

\subsubsection{Rapid detection of carbapenemase activity by RAPIDEC CARBA NP test.}

This assay was done as instructed by the manufacturer (bioMèrieux SAMarcy 'Etoile France, Lot No. 1005258372). Briefly, a loop full of bacterial colonies was taken from an overnight-incubated Muller Hinton agar Petri dish then mixed with API suspension medium given with the kit. Then the microbial suspensions were inoculated into wells in the provided test stripe and then incubated at $37^{\circ} \mathrm{C}$. Reading of the results was carried on within 30- $40 \mathrm{~min}$. A negative result was indicated by a red color, while a positive test showed a color change from red to yellow orange.

\subsubsection{Modified Carbapenem Inactivation Method (mCIM)}

A suspension was prepared using one loop full of each isolate in $2 \mathrm{ml}$ tryptic soy broth (Oxoid, England). Then, a sensitivity testing disk containing $10 \mu \mathrm{g}$ meropenem (Oxoid, England) was placed in the bacterial suspension and then incubated for 4 hours at $37^{\circ} \mathrm{C}$. Then the disk was removed from the bacterial suspension and placed on an agar plate of Mueller Hinton that was inoculated with a susceptible E. coli indicator strain (ATCC 25922) followed by incubation at $37^{\circ} \mathrm{C}$. If the bacterial isolate produced carbapenemase, then the meropenem was inactivated while the carbapenemase nonproducing isolate yielded a clear inhibition zone [10].

\subsection{Carbapenemase genes detection by PCR:}

\subsubsection{Extraction of DNA:}

DNA extraction was done by heat shock technique in which fresh culture of the test organism was suspended in $200 \mathrm{ml}$ of sterilized distilled $\mathrm{H} 2 \mathrm{O}$ and mixed by a vortex to acquire a homogenous suspension. Then the cells were lysed by incubation in a water bath at $100^{\circ} \mathrm{C}$ for seven minutes followed by placing in ice for two minutes. Then centrifugation at $10000 \mathrm{rpm}$ was done for five minutes for removal of cellular debris. Finally, the supernatant containing the DNA was aspirated and stored at $-70^{\circ} \mathrm{C}$ to be used for amplification [13].

\subsubsection{PCR amplification:}

Amplification of blaKPC, blaNDM, blaIMP, and blaOXA-48 genes was performed by PCR technique. The total reaction volume was $25 \mu$ l containing 8.5 $\mu \mathrm{l}$ distilled water, $1 \mu \mathrm{l}$ of each primer (Invitrogen, Germany) at a concentration 
of 20 picomolar, $12.5 \mu$ of Taq Master Mix Kit (QIAGEN Cat. No.201203), and $2 \mu 1$ of the extracted DNA. Primers were prepared according to manufacturer's instructions. The specific forward and reverse primers for the amplification of blaKPC, blaNDM, blaIMP and blaOXA-48 genes are presented in Table. 1 .

\subsection{Statistical analysis:}

The data were input in Microsoft Excel program, analyzed by SPSS (version 18) and the results were presented in charts and tables. Chi-square test was used to compare proportions among two qualitative parameters.

\section{Results}

The distribution of CRKP and CSKP in relation to the type of clinical specimens and the antimicrobial sensitivity pattern of the studied isolates are described in Table. 2 and Table. 3 respectively.

\subsection{Phenotypic and Genotypic detection of carbapenemases:}

Rapidec Carba NP test, mCIM and PCR were performed on the 60 isolates. Carbapenemase-producers were 27 (45\%) isolates by Rapidec Carba NP test and 20 $(33.3 \%)$ by mCIM test while $24(40 \%)$ isolates were positive by PCR for carbapenemase genes as shown in Table. 4 and Fig.1.

Among the 24 PCR positive isolates, one $(4.1 \%)$ isolate had blaKPC gene, $13(54.2 \%)$ isolates were positive for blaNDM, 7 (29.2\%) of isolates were positive for blaOXA-48 and 3 $(12.5 \%)$ isolates were positive for both blaNDM and blaOXA48. All isolates were negative for blaIMP. The performance of phenotypic tests (Rapidec test, mCIM) in comparison to PCR is demonstrated in Table. 5 .

Table (1): The primer sequences used for carbapenemase gene detection by PCR Al-Zahrani and Alasiri (2018) and Zarakolu et al., 2016 [14,15].

\begin{tabular}{|c|c|r|c|}
\hline \multirow{2}{*}{ Gene } & Primer & Amplicon \\
blaKPC & KPC F & 5'-sequence-3' & size \\
\cline { 2 - 3 } & KPC R & 5'-CTTGTCATCCTTGTTAGGCG-3' & \multirow{2}{*}{$798 \mathrm{bp}$} \\
\hline \multirow{2}{*}{ blaNDM } & NDM F & 5'-GGGCAGTCGCTTCCAACGGT-3' & \multirow{2}{*}{$475 \mathrm{bp}$} \\
\cline { 2 - 3 } & NDM R & 5'-GTAGTGCTCAGTGTCGGC-3' & \\
\hline \multirow{2}{*}{ blaOXA-48 } & OXA-48 F & 5'-TGTTTTTGGTGGCATCGAT-3' & \multirow{2}{*}{$300 \mathrm{bp}$} \\
\cline { 2 - 3 } & OXA-48 R & 5'-GATCGCGATTCCAAGTGG-3' & \\
\hline \multirow{2}{*}{ blaIMP } & IMP-F & 5'GGAATAGAGTGGCTTAAYTCTC-3' & \multirow{2}{*}{$188 \mathrm{bp}$} \\
\cline { 2 - 3 } & IMP-R & 5'-CCAAACYACTASGTTATCT-3' & \\
\hline
\end{tabular}


Table (2): The distribution of CRKP and CSKP in relation to the type of clinical specimens. $C S=$ Carbapenem sensitive, $C R=$ Carbapenem resistant, $S=$ Significant $(P$ value $<0.05), N S=$ nonsignificant ( $p$ value >0.05).

\begin{tabular}{|c|c|c|c|c|c|c|}
\hline & Total isolates & No. \& \%of CS & No. \& \% of CR & \multirow{2}{*}{$\begin{array}{l}\text { Test } \\
\text { value }\end{array}$} & \multirow{2}{*}{ P-value } & \multirow{2}{*}{ Sig. } \\
\hline & No. $=60$ & No. $=32$ & No. $=28$ & & & \\
\hline Urine & $41(68.3 \%)$ & $18(56.3 \%)$ & $23(82.1 \%)$ & 4.627 & 0.031 & $S$ \\
\hline Sputum & $10(16.7 \%)$ & $6(18.8 \%)$ & $4(14.3 \%)$ & 0.214 & 0.643 & NS \\
\hline Wound swab & $4(6.7 \%)$ & $3(9.4 \%)$ & $1(3.6 \%)$ & 0.808 & 0.368 & NS \\
\hline Pus swabs & $2(3.3 \%)$ & $2(6.3 \%)$ & $0(0.0 \%)$ & 1.810 & 0.178 & NS \\
\hline Blood & $1(1.7 \%)$ & $1(3.1 \%)$ & $0(0.0 \%)$ & 0.890 & 0.345 & NS \\
\hline $\begin{array}{l}\text { Peritoneal } \\
\text { fluid }\end{array}$ & $2(3.3 \%)$ & $2(6.3 \%)$ & $0(0.0 \%)$ & 1.810 & 0.178 & NS \\
\hline
\end{tabular}

Table (3): Antimicrobial resistance pattern of the studied K. pneumoniae isolates. ESBL: Extended spectrum beta lactamases CSKP $=$ Carbapenem-sensitive Klebsiella pneumonia, CRKP= Carbapenem-resistant Klebsiella pneumonia. MDR: multidrug resistant, ampC: ampler class $C$.

\begin{tabular}{|c|c|c|c|c|}
\hline Resistance & CRKP (28) & CSKP (32) & P value & Significance \\
\hline ESBL & $26(92.9 \%)$ & $12(37.5 \%)$ & 19.705 & $<0.001$ \\
\hline ampC & $4(14.3 \%)$ & ----- & 4.898 & 0.027 \\
\hline Ciprofloxacin & $28(100 \%)$ & $18(56.3 \%)$ & 12.857 & $<0.001$ \\
\hline Norfloxacin & $27(96.4 \%)$ & $10(31.3 \%)$ & 27.265 & $<0.001$ \\
\hline Gentamicin & $25(89.3 \%)$ & $7(21.9 \%)$ & 23.907 & $<0.001$ \\
\hline Amikacin & $22(78.6 \%)$ & $5(15.6 \%)$ & 29.921 & $<0.001$ \\
\hline MDR & $28(100 \%)$ & $15(46.9 \%)$ & 14.725 & $<0.001$ \\
\hline
\end{tabular}


Table (4): Detection of carbapenemase production among the studied K. pneumoniae isolates by Rapidec carba NP test, $\mathrm{mCIM}$ test and PCR. CRKP=Carbapenem-resistant $k$. pneumonia, CSKP= Carbapenem-sensitive $k$. pneumoniae, $m$ CIM=modified carbapenem inhibition method.

\begin{tabular}{|c|c|c|c|c|c|c|}
\hline \multirow{2}{*}{ Tested } & \multicolumn{2}{|c|}{ Rapidec Carba NP } & \multicolumn{2}{c|}{ mCIM } & \multicolumn{2}{c|}{ PCR } \\
\cline { 2 - 7 } & Positive & negative & positive & negative & positive & negative \\
\hline CRKP (28) & $27(96.4 \%)$ & $1(3.6 \%)$ & $20(71.4 \%)$ & $8(28.6)$ & $24(85.7 \%)$ & $4(14.3 \%)$ \\
\hline CSKP (32) & 0 & $32(100 \%)$ & 0 & $32(100 \%)$ & 0 & $32(100 \%)$ \\
\hline Total (60) & 27 & 33 & 20 & 40 & 24 & 36 \\
\hline
\end{tabular}

Table (5): The performance of phenotypic tests (Rapidec test, $\mathrm{mCIM}$ ) for detection of carbapenemase genes in comparison to $\mathrm{PCR}$. $T N=$ true negative, $T P=$ true positive, $F N=$ false negative, $F P=$ false positive, $N P V=$ negative predictive value $=P P V$, positive predictive value, $m C I M=$ modified carbapenem inhibition method

\begin{tabular}{|c|c|c|c|c|c|c|c|c|c|}
\hline Parameter & TP & TN & FP & FN & Accuracy & Sensitivity & Specificity & PPV & NPV \\
\hline Rapidec Carba NP & 24 & 32 & 4 & 0 & $95 \%$ & $100 \%$ & $91.7 \%$ & $88.8 \%$ & $100 \%$ \\
\hline mCIM & 16 & 32 & 4 & 8 & $80 \%$ & $66.7 \%$ & $88.9 \%$ & $80 \%$ & $80 \%$ \\
\hline
\end{tabular}

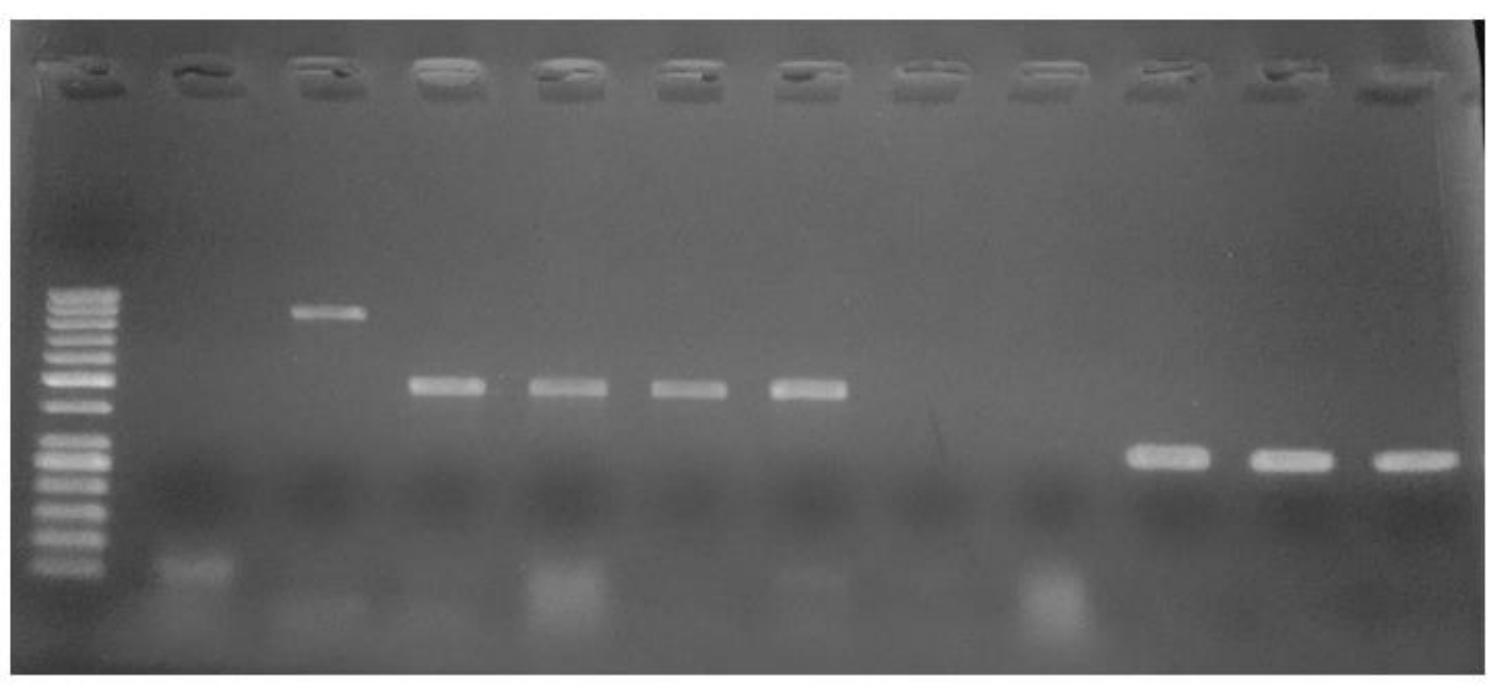

Figure (1): Carbapenemase genes detection in K. pneumoniae isolates by PCR. Lane 1: molecular weight ladder (50 bp), Lane 2: control negative, Lane 3: blaKPC positive isolate (798 bp), Lanes 4, 5, 6 and 7: blaNDM positive isolates (475 bp) Lanes 8 and 9: negative isolates, Lane 10, 11 and 12: blaOXA-48 positive isolates ( $300 \mathrm{bp}$ ). 


\section{Discussion}

Carbapenemase- producing $K$ pneumonia (CPKP) has been an important issue in terms of public health and clinical outcome as it is usually resistant to almost all antimicrobial agents and due to its rapid worldwide spread [16,17]. The CRKP prevalence is variable between countries and even between medical health care settings in the same country. In our study, $46.7 \%$ (28/60) of isolated k. pneumoniae were carbapenem-resistant.

Carbapenem resistance in Egypt, is alarming, as it was identified in $44.3 \%$ of $K$. pneumoniae isolates in the hospitals of Suez Canal University, which is similar to its prevalence in our hospital [18]. However, lower prevalence rates of $(33 \%)$ and $(13.9 \%)$ were reported at Mansoura university hospitals and at the Egyptian National Cancer Institute respectively $[19,20]$. The high frequency of CRKP in this study may be due to the frequent use of carbapenems empirically at our hospital.

In the present study, carbapenem-resistant $K$ pneumoniae were more prevalent in urine (82\%), followed by sputum specimens (14\%). Li et al., 2019 reported that $K$ pneumoniae was isolated from respiratory specimens $(62.9 \%)$ followed by blood [21]. However, they found no significant differences between CRKP and CSKP as regard the source of samples. In an Egyptian study, respiratory specimens $(62 \%)$ were the major source of CRKP, followed by urine samples (14\%) [19]. All 28 CRKP isolates in the present study were MDR, 93\% were ESBL producers, while $14.3 \%$ were ampC producers. This significant association of carbapenemresistance with multidrug resistance among our $K$. pneumoniae studied isolates was also reported in several studies. Li et al., 2019 demonstrated that the tested antibiotics in the group of CSKP showed better sensitivity (65\%) than in CRKP, except for ampicillin-sulbactam as it was
(55.9\%) while for piperacillin it was $(55.9 \%)$ [21]. It was reported that carbapenem-resistant isolates often carry more resistance determinants and shows complex phenotypes of MDR. Moreover, higher-level of carbapenem-resistance are regularly accompanied with expression of mobile genetic elements within carbapenemase genes [22].

Reliable and rapid identification of carbapenemases is crucial for development of infection control strategies. To date, phenotypic methods are the tools available in the routine Microbiology laboratory. In this study, we assessed the performance of two phenotypic techniques for detection of carbapenemase in K. pneumoniae and their applicability in the clinical Microbiology laboratory. The RAPIDEC® CARBA NP assay is a fast phenotypic test that does not require expensive devices or special expertise, furthermore, it is considered to have a low cost in relation to molecular tests [22].

This study revealed that all isolates of $K$. pneumoniae carrying carbapenemase genes were detected by Rapidec carba NP test with $(100 \%)$ sensitivity. This was in agreement with results obtained by Dortet et al. (2015) who reported a sensitivity of $99 \%$ among Enterobacteriaceae isolates [23].

Lower sensitivities were reported in several studies [6, 22, 24, 25]. However, in these studies the lower sensitivities were attributed to greater proportion of class D producing A. baumannii complex among the tested isolates which were not included in our research and when the sensitivity was calculated only for Enterobacteriaceae isolates, it raised from $70 \%$ to $100 \%$ [22]. In this study, the Rapidec Carba NP test specificity was $91.7 \%$. Higher specificities (98\% and $100 \%$ ) were reported in other studies [22,7]. However, the false-positive results observed in our study were also reported elsewhere $[6,26,27]$, and may be 
attributed to a decreased stability of the imipenem substrate toward strains producing enzymes with weak carbapenemase activity but are not true carbapenemases (e.g., AmpCs, CMY, CTX-M-type producers) and may be due to using the recommended inoculum in the Producer's directives. Dortet et al. (2015) discovered a serious effect of the bacterial inoculum in the test performance and suggested using a greater amount of the inoculum to decrease false positive results [23].

The mCIM is a straightforward and costeffective test available in many clinical laboratories and is recommended by CLSI 2018 for detection of carbapenemases. The present study showed that the sensitivity and specificity of this assay for detection of carbapenemase production in $K$. pneumonia were $(66.7 \%)$ and $(88.9 \%)$ respectively. The test failed to detect the blaKPC producing isolate and all blaNDM positive isolates.

Better performance of mCIM was achieved in other studies, (Aktaş et al., 2017) reported $100 \%$ sensitivity and specificity when evaluating the test using Enterobacteriaceae isolates [7]. Tijet et al., 2015 compared the performance of both carba NP test and $\mathrm{mCIM}$ and reported better sensitivity and specificity of mCIM which is inconsistent with our results [28]. However, the authors in that study used the carba NP test and not our commercially available Rapidec Carba NP test which may have better performance.

In our study, again, the four isolates that were falsely positive by Rapidec carpa NP test, were falsely positive upon testing with mCIM. In a study by (Jing et al., 2018), false positive result in a $K$. pneumoniae strain making CTX-15 was reported [8]. Similarly, Pierce et al. (2017) stated that TEM-1 and TEM-52 enzymes may produce false positive results in mCIM tests [29]. Molecular techniques remain the gold standard that are reliable for detection of carbapenemases-producing bacteria more than phenotypic methods. However, they have some limitations as the results may be false-negative due to mutations affecting annealing of primers or the occurrence of a carbapenemase gene that is not tested for in the PCR test or it may give false positive results as in cases of detecting silent genes (i.e. no expression of carbapenemase) [25]. In the present study, carbapenemase coding genes in $K$. pneumonia isolates were detected in 24 out of the $28(85.7 \%)$ carbapenem resistant isolates, while in the four isolates none of the tested carbapenemase genes (blaIMP, blaKPC, blaOXA-48, and blaNDM) were found. These four isolates were positive by both Rapidec carba NP and mCIM methods. Carbapenem- resistance shown in these isolates may be due to a presence of both ESBLs and outer membrane proteins changes (ESBL/Omp) or may be mediated by other carbapenemase genes that were not tested in our study.

This is the first study that reported the frequency and distribution pattern of carbapenemase genes in CRKP clinical isolates in our hospital. In our study, the most prevalent gene among the 24 carbapenemase positive $K$. pneumoniae isolates was blaNDM 13/24 (54.2) \% which differs from a previous study in Egypt, where blaOXA-48-like types were the most predominant $(28.6 \%)$ while $2.4 \%$ only of CRKP carried bla NDM [30]. In another Egyptian study, the most prevalent gene among the 42 CRKP isolates tested was blaKPC at $43.5 \%$ while blaNDM-1 genes was detected in only $4.3 \%$ [19].

In our study, one (1.6\%) isolate of $K$. pneumoniae, identified as blaKPC producer. This low frequency was also reported in Saudi Arabia, where no blaKPC producers were detected among isolates investigated [14]. While a much higher prevalence $(89 \%)$ was reported in another study [31].

In the present study, the blaOXA-48 producer's prevalence among CRKP was $29.2 \% \quad(7 / 24)$. While other studies identified blaOXA48 as the most prevalent carbapenemase [32]. Also, a much higher 
prevalence was reported by (Al-Zahrani and Alasiri, 2018) who reported that $(81.5 \%)$ isolates of $K$. pneumoniae were harboring blaOXA-48 [14].

The presence of several carbapenemase genes in the same isolate raises the concern about the likelihood of multiple carbapenemase genes crossing onto the same plasmid. This study detected three isolates (12.5\%) that were positive for both blaNDM and blaOXA-48. Another study showed that the first isolate of $K$. pneumoniae carring both blaOXA-48 and blaNDM-1 in Turkey was isolated from a patient living nearby the border of Syria [33]. Dual carbapenemase prevalence was fortunately lower $(1.5 \%)$ in another study [34].

\section{Conclusion:}

In conclusion, both Rapidec carba NP and mCIM tests have a high performance and applicability in the carbapenemases detection. The Rapidec carba NP is a simple rapid test with high sensitivity but at a low cost than molecular methods. However, the mCIM method also represents a simple valuable tool for district

\section{References}

1- Chen D, Hu X, Chen F, Li H, Wang D, Li X, et al. (2019). Co-outbreak of multidrug resistance and a novel ST3006 Klebsiella pneumoniae in a neonatal intensive care unit: A retrospective study. Medicine;98(4).

2- Pan H, Lou Y, Zeng L, Wang L, Zhang J, Yu W, et al. (2019). Infections Caused by Carbapenemase-Producing Klebsiella pneumoniae: Microbiological Characteristics and Risk Factors. Microbial Drug Resistance; 25(2):287-96. microbiology laboratories especially in low-income countries due to its simplicity, availability and cost effectiveness.

High rate of resistance to carbapenems is found in K. pneumoniae clinical isolates in our hospital. The most prevalent gene is blaNDM, and dual carbapenemase genes were detected, these findings necessitate the immediate implementation of antibiotic stewardship program and infection control strategies.

Further studies for profiling of all resistance gene determinants in the CRKP isolates is recommended to direct a proper antibiotic policy.

The authors declare that they do not have any conflict of interest and they do not receive any fund for this work.

Funding: This work didn't receive any fund from any authority and totally funded by the authors.

Conflict of interest: Authors have nothing to disclose.

3- Djahmi N, Dunyach-Remy C, Pantel A, Dekhil M, Sotto A, Lavigne JP. (2014). Epidemiology of carbapenemase-producing Enterobacteriaceae and Acinetobacter baumannii in Mediterranean countries. BioMed research international; 2014.

4- Walker CD, Shankaran S. (2016). Extended antibiotic resistance in carbapenemase-producing

Klebsiella pneumoniae: A case series. American journal of infection control; 44(9):1050-2.

5- Monaco M, Giani T, Raffone M, Arena F, Garcia-Fernandez A, Pollini S, et al. (2014). Colistin resistance superimposed to endemic 
carbapenem-resistant Klebsiella pneumoniae: a rapidly evolving problem in Italy, November 2013 to April 2014. Euro surveillance; 19 (42):20939.

6- Poirel L, Nordmann P. (2015). Rapidec Carba NP test for rapid detection of carbapenemase producers. Journal of clinical microbiology; 53(9):3003-8.

7- Aktaş E, Malkoçoğlu G, Otlu B, Çopur Çiçek A, Külah C, Cömert F, et al. (2017). Evaluation of the carbapenem inactivation method for detection of carbapenemaseproducing Gram-negative bacteria in comparison with the RAPIDEC CARBA NP. Microbial drug resistance; 23(4):457-61.

8- Jing $X$, Zhou H, Min X, Zhang X, Yang Q, Du S, et al. (2018). The simplified carbapenem inactivation method (sCIM) for simple and accurate detection of carbapenemase-producing Gramnegative bacilli. Frontiers in microbiology; 9:2391.

9- Hombach M, von Gunten B, Castelberg C, Bloemberg GV. (2015). Evaluation of the Rapidec Carba NP test for detection of carbapenemases in Enterobacteriaceae. Journal of clinical microbiology; 53(12):3828-33.

10-Clinical and Laboratory Standards Institute. (2018). Performance standards for antimicrobial susceptibility testing; twenty-eighth informational supplement Document M100-S28. Wayne, PA: CLSI; 2018.
11-Chowdhury AH, Nandi S, Rahman M, Karim AA, Mamtaz SS, Ara NN, et al. (2016). Comparison Between Phenotypic Confirmatory Test \& Double Disc Synergy Test in Detection of Extended Spectrum bLactamases Producers Among Gram-Negative Bacilli. Chattagram Maa-O-Shishu Hospital Medical College Journal; 15 (2):3-8.

12-Gupta G, Tak V, Mathur P. (2014). Detection of AmpC $\beta$ Lactamases in Gram-negative Bacteria. J Lab Physicians; 6 (1):1-6.

13- Manoharan A, Chatterjee S, Mathai D, SARI Study Group. (2010). Detection and characterization of metallo beta lactamases producing Pseudomonas aeruginosa. Indian Journal of Medical Microbiology; $28(3): 241$.

14-Al-Zahrani I. A, Alasiri B A. (2018). The emergence of carbapenem-resistant Klebsiella pneumoniae isolates producing OXA-48 and NDM in the Southern (Asir) province, Saudi Arabia Saudi Med J; 39 (1): 23-30.

15-Zarakolu P, Eser OK, Aladag E, AlZahrani IA, Day KM, Atmaca O, et al. (2016). Epidemiology of carbapenem-resistant Klebsiella pneumoniae colonization: a surveillance study at a Turkish university hospital from 2009 to 2013. Diagnostic microbiology and infectious disease; 85(4):466-70.

16- Patel G, Bonomo R. (2013). "Stormy waters ahead": global emergence of carbapenemases. Frontiers in microbiology; 4:48. 
17- Saidel-Odes L, Borer A. (2014). Limiting and controlling carbapenem-resistant Klebsiella pneumoniae. Infection and drug resistance; 7:9.

18-El-Sweify MA, Gomaa NI, ElMaraghy NN, Mohamed HA. (2015). Phenotypic Detection of Carbapenem Resistance among Klebsiella pneumoniae in Suez Canal University Hospitals, Ismailiya, Egypt. Egypt Int J Curr Microbiol App Sci; 4:10-8.

19- Moemen D, Masallat DT. (2017). Prevalence and characterization of carbapenem-resistant Klebsiella pneumoniae isolated from intensive care units of Mansoura University hospitals. Egyptian Journal of Basic and Applied Sciences; 4 (1):37-41.

20- Ashour HM, El-Sharif A. (2009). Species distribution and antimicrobial susceptibility of gram-negative aerobic bacteria in hospitalized cancer patients. Journal of translational medicine; 7 (1): 14 .

21-Li Y, Shen H, Zhu C, Yu Y. (2019): Carbapenem-Resistant Klebsiella pneumoniae Infections among ICU Admission Patients in Central China: Prevalence and Prediction Model. BioMed research international; 2019.

22-Coppi M, Antonelli A, Giani T, Spanu T, Liotti FM, Fontana C, et al. (2017). Multicenter evaluation of the RAPIDEC@ CARBA NP test for rapid screening of carbapenemase-producing Enterobacteriaceae and Gramnegative nonfermenters from clinical specimens. Diagnostic microbiology and infectious disease; 88 (3):207-13.

23-Dortet L, Agathine A, Naas T, Cuzon G, Poirel L, Nordmann P. (2015). Evaluation of the RAPIDEC $®$ CARBA NP, the Rapid CARB Screen ${ }^{\circledR}$ and the Carba NP test for biochemical detection of carbapenemaseproducing Enterobacteriaceae. Journal of Antimicrobial Chemotherapy; 70 (11):3014-22.

24-Garg A, Garg J, Upadhyay GC, Agarwal A, Bhattacharjee A. (2015). Evaluation of the Rapidec Carba NP test kit for detection of carbapenemase-producing Gramnegative bacteria. Antimicrobial agents and chemotherapy; 59 (12):7870-2.

25-Kabir MH, Meunier D, Hopkins KL, Giske CG, Woodford N. (2016). A two-centre evaluation of RAPIDEC $\AA$ CARBA NP for carbapenemase detection in Enterobacteriaceae, Pseudomonas aeruginosa and Acinetobacter spp. Journal of Antimicrobial Chemotherapy; 71 (5):1213-6.

26-Lifshitz Z, Adler A, Carmeli Y. (2016). Comparative study of a novel biochemical assay, the Rapidec Carba NP test, for detecting carbapenemaseproducing Enterobacteriaceae. Journal of clinical microbiology; 54 (2):453-6.

27-Österblad M, Lindholm L, Jalava J. (2016). Evaluation of two commercial carbapenemase gene assays, the Rapidec Carba NP test and the in-house Rapid Carba NP test, on bacterial cultures. Journal of 
Antimicrobial Chemotherapy; 71 (7):2057-9.

28-Tijet N, Patel SN, Melano RG. (2015). Detection of carbapenemase activity in Enterobacteriaceae: comparison of the carbapenem inactivation method versus the Carba NP test. Journal of Antimicrobial Chemotherapy; 71 (1):274-6.

29- Pierce VM, Simner PJ, Lonsway DR, Roe-Carpenter DE, Johnson JK, Brasso WB, et al. (2017). The modified carbapenem inactivation Method (mCIM) for phenotypic detection of carbapenemase production among Enterobacteriaceae. J. Clin. Microbiol.; 55: 2321-2333.

30-Morsi SS. (2016). Comparative Evaluation of Phenotypic and Genotypic Methods for Detection of Carbapenemases in Clinically Significant Klebsiella pneumoniae Isolates. The Egyptian Journal of Medical Microbiology; 38 (87):1-8.

31- Logan LK, Nguyen DC, Huang FA, Qureshi NK, Charnot-Katsikas A, Bartlett AH, et al. (2019). A MultiCentered Case-Case-Control Study of Factors Associated with Klebsiella pneumoniae Carbapenemase-Producing Enterobacteriaceae Infections in Children and Young Adults. The Pediatric infectious disease journal; 38 (5):490-5.

32-Brañas P, Villa J, Viedma E, Mingorance J, Orellana MA, Chaves F. (2015). Molecular epidemiology of carbapenemaseproducing Klebsiella pneumoniae in a hospital in Madrid: successful establishment of an OXA-48 ST11 clone. International journal of antimicrobial agents; 46 (1):111-6.

33-Kilic A, Baysallar M. (2015). The first Klebsiella pneumoniae isolate co-producing OXA-48 and NDM-1 in Turkey. Annals of laboratory medicine; 35 (3):382-3.

34-Biedenbach D, Bouchillon S, Hackel M, Hoban D, Kazmierczak K, Hawser S, et al. (2015). Dissemination of NDM metallo- $\beta$ lactamase genes among clinical isolates of Enterobacteriaceae collected during the SMART global surveillance study from 2008 to 2012. Antimicrobial agents and chemotherapy; 59 (2):826-30. 